\title{
$\mathrm{C}_{1}$-symmetry pathway
}

\section{Levels of calculation}

Geometries (S1 state): SA-3-CASSCF $(12,11) / 6-31 \mathrm{G}(\mathrm{d}, \mathrm{p})$

Energies: SA-5-CASSCF $(12,12)$ and MS-5-CASPT2 $(12,12)$ with the $6-31++\mathrm{G}(\mathrm{d}, \mathrm{p})$ and $6-31(+) \mathrm{G}(\mathrm{d}, \mathrm{p})$ basis sets

Cartesian coordinates are given in Bohr and energies in a.u.

\section{ENOL}

Cartesian coordinates (Bohr)

\begin{tabular}{|c|c|c|c|c|}
\hline & \multicolumn{3}{|c|}{ No. Label } \\
\hline & $\mathrm{C} 1$ & & & \\
\hline & $\mathrm{C} 2$ & & 9977 & \\
\hline & C3 & & & -0.1754 \\
\hline & $\mathrm{C} 4$ & & & \\
\hline 5 & C5 & & & \\
\hline 6 & C6 & -0.49 & & 01959 \\
\hline & C7 & & & \\
\hline$x$ & C8 & & & \\
\hline 9 & C9 & & -4.26398 & \\
\hline 0 & O1 & & & \\
\hline & N1 & & & \\
\hline & N2 & -7.31 & 2.60723 & 0.9300 \\
\hline & N3 & & & \\
\hline & $\mathrm{N} 4$ & & -6.4 & 0.7 \\
\hline 5 & H1 & -2.31 & & 0.36877 \\
\hline 16 & $\mathrm{H} 2$ & & & \\
\hline 7 & $\mathrm{H} 3$ & & & -0 \\
\hline 18 & $\mathrm{H} 4$ & & $0.4 \mathrm{C}$ & -0 \\
\hline 9 & H5 & & & \\
\hline 0 & H6 & & -6.28 & 0.32785 \\
\hline 21 & $\mathrm{H} 7$ & -4.1( & 4.09 & 0.35655 \\
\hline 2 & $\mathrm{H} 8$ & & & \\
\hline 23 & H9 & -7.67218 & 2.45438 & 2.78250 \\
\hline 24 & H10 & & & 0.21607 \\
\hline 5 & H11 & & & \\
\hline 26 & H12 & 51 & -4.00 & -0.7727 \\
\hline 21 & H13 & & & \\
\hline & H14 & & & \\
\hline 29 & H15 & -2.124 & -5.30549 & $\begin{array}{ll}9 & 0.55\end{array}$ \\
\hline 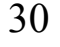 & & & & \\
\hline
\end{tabular}

Nuclear repulsion energy $=849.690878$

SA-5-CASSCF(12,11)/6-31++G(d,p) energies: 
root number $1 \mathrm{E}=\quad-642.98307717$ a.u.

root number $2 \mathrm{E}=-642.84594737$ a.u.

root number $3 \mathrm{E}=-642.79270717$ a.u.

root number $4 \mathrm{E}=-642.77372604$ a.u.

root number $5 \mathrm{E}=-642.75846957$ a.u.

MS-5-CASPT2/6-31++G(d,p) energies:

$\begin{array}{llllll}-644.95413802 & -644.83089832 & -644.79380279 & -644.75369210 & -644.74608648\end{array}$

\section{SA-5-CASSCF(12,11)/6-31(+)G(d,p) energies:}

root number $1 \mathrm{E}=\quad-642.97133338$ a.u.

root number $2 \mathrm{E}=-642.83409336$ a.u.

root number $3 \mathrm{E}=-642.77454126$ a.u.

root number $4 \mathrm{E}=-642.75906033$ a.u.

root number $5 \mathrm{E}=-642.74397063$ a.u.

MS-5-CASPT2/6-31(+)G(d,p) energies:

$\begin{array}{llllll}-644.92543250 & -644.80087319 & -644.76894264 & -644.71928018 & -644.71523007\end{array}$

PT1 (optimization led to ENOL; see the manuscript)

Cartesian coordinates:

\begin{tabular}{|c|c|c|c|c|}
\hline No. & - Label & X & Y & \\
\hline 1 & $\mathrm{C} 1$ & & -0 & \\
\hline 2 & $\mathrm{C} 2$ & & & -0.18548 \\
\hline 3 & $\mathrm{C} 3$ & 70 & 5165 & -0.31804 \\
\hline 4 & $\mathrm{C} 4$ & 16652 & -2.79940 & -0.11095 \\
\hline 5 & C5 & & -4 & 797 \\
\hline 6 & C6 & & 2319 & 5940 \\
\hline 7 & C7 & 1.043 & 4.93777 & -0.24457 \\
\hline 8 & $\mathrm{C} 8$ & -1.15 & 3.4 & 706 \\
\hline 9 & C9 & 3 & 0.7 & 705 \\
\hline 10 & $\mathrm{O}$ & 7 & 4.3 & 3427 \\
\hline 11 & N1 & 24 & -3.04886 & $5 \quad 0.34114$ \\
\hline 12 & N2 & 3 & 1.73673 & 1.05010 \\
\hline 13 & N3 & -7.506 & -2.87289 & -1.6879 \\
\hline 14 & N4 & -3.4 & -6.29121 & 0.75666 \\
\hline 15 & H1 & -2.51300 & -0.44043 & 30.5406 \\
\hline 16 & $\mathrm{H} 2$ & 5.048 & 4.84972 & -0.62244 \\
\hline 17 & $\mathrm{H} 3$ & & 6.95012 & -0.3881 \\
\hline 18 & H4 & 80754 & 0.93058 & -0.56653 \\
\hline 19 & H5 & 4 & -3.80052 & -0.1974 \\
\hline 20 & H6 & & -6.18489 & 0.3671 \\
\hline 21 & $\mathrm{H} 7$ & -5.72134 & 2.71718 & 0.67013 \\
\hline 22 & H8 & -7.53651 & 0.05619 & 0.0226 \\
\hline
\end{tabular}


$\begin{array}{lllll}23 & \text { H9 } & -7.52227 & 1.34654 & 2.90614\end{array}$

$\begin{array}{lllll}24 & \mathrm{H} 10 & -8.89857 & 2.86187 & 0.59617\end{array}$

$\begin{array}{lllll}25 & \mathrm{H} 11 & -6.87527 & -2.58696 & -3.45291\end{array}$

$\begin{array}{llllll}26 & \mathrm{H} 12 & -6.23634 & -4.02702 & -0.83076\end{array}$

$\begin{array}{llllll}27 & \mathrm{H} 13 & -9.13258 & -3.83498 & -1.83671\end{array}$

$\begin{array}{lllll}28 & \mathrm{H} 14 & -3.17206 & -7.85152 & -0.29033\end{array}$

$\begin{array}{lllll}29 & \mathrm{H} 15 & -1.81292 & -5.26005 & 0.60101\end{array}$

$\begin{array}{lllll}30 & \mathrm{H} 16 & -3.52851 & -6.86736 & 2.55766\end{array}$

Nuclear repulsion energy $=868.578931$

\section{SA-5-CASSCF $(12,11) / 6-31++G(d, p)$ energies:}

root number $1 \mathrm{E}=-642.92993298$ a.u.

root number $2 \mathrm{E}=-642.83266279$ a.u.

root number $3 \mathrm{E}=-642.81247608$ a.u.

root number $4 \mathrm{E}=\quad-642.76030531$ a.u.

root number $5 \mathrm{E}=\quad-642.75432745$ a.u.

MS-5-CASPT2/6-31++G(d,p) energies:

$\begin{array}{llllll}-644.92900953 & -644.83261710 & -644.80268809 & -644.77803524 & -644.74161733\end{array}$

\section{SA-5-CASSCF(12,11)/6-31(+)G(d,p) energies:}

root number $1 \mathrm{E}=-642.91034859$ a.u.

root number $2 \mathrm{E}=-642.82480907$ a.u.

root number $3 \mathrm{E}=\quad-642.79312359$ a.u.

root number $4 \mathrm{E}=\quad-642.75166717$ a.u.

root number $5 \mathrm{E}=-642.73488203$ a.u.

MS-5-CASPT2/6-31(+)G(d,p) energies:

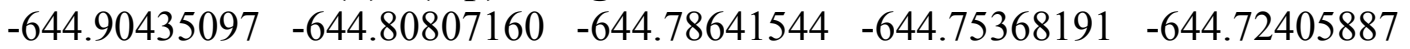

\section{TS $_{\mathbf{P T}}$}

Cartesian coordinates:

\begin{tabular}{|c|c|c|c|}
\hline No. Label & $\mathrm{X}$ & $\mathrm{Y}$ & Z \\
\hline $\mathrm{C} 1$ & 1.09 & 0 & -1.1 \\
\hline $\mathrm{C} 2$ & & -0 . & $5-0$. \\
\hline 3 C3 & -3.0 & 745 & 1071 \\
\hline $4 \mathrm{C} 4$ & -2.45 & 4.40487 & -0.10504 \\
\hline $5 \mathrm{C} 5$ & -0.12 & 5.25291 & -0.9 \\
\hline 6 C6 & 1.78 & 3.40 & -1.43269 \\
\hline $7 \quad \mathrm{C} 7$ & -5.502 & 0.98919 & 1.25815 \\
\hline $8 \mathrm{C} 8$ & -5.813 & -1.614 & 1.66823 \\
\hline 9 C9 & -3.84949 & -3.29826 & \\
\hline $10 \mathrm{O} 1$ & 4.03089 & 3.92796 & $6-1.9783$ \\
\hline
\end{tabular}




$\begin{array}{llrrr}11 & \mathrm{~N} 1 & -1.53506 & -2.56176 & 0.22537 \\ 12 & \mathrm{~N} 2 & 6.95030 & 0.43954 & 1.06931 \\ 13 & \mathrm{~N} 3 & 4.16528 & -3.44447 & 2.18686 \\ 14 & \mathrm{~N} 4 & 2.47842 & -6.51942 & -2.32314 \\ 15 & \mathrm{H} 1 & 2.46277 & -0.60221 & -1.80094 \\ 16 & \mathrm{H} 2 & -3.90117 & 5.78345 & 0.30874 \\ 17 & \mathrm{H} 3 & 0.30695 & 7.22291 & -1.14250 \\ 18 & \mathrm{H} 4 & -6.98939 & 2.31580 & 1.64767 \\ 19 & \mathrm{H} 5 & -7.57573 & -2.33928 & 2.38119 \\ 20 & \mathrm{H} 6 & -4.08841 & -5.29066 & 1.48853 \\ 21 & \mathrm{H} 7 & 6.05473 & 1.55896 & -0.22296 \\ 22 & \mathrm{H} 8 & 5.62688 & -1.41322 & 1.63516 \\ 23 & \mathrm{H} 9 & 8.62080 & -0.09566 & 0.34531 \\ 24 & \mathrm{H} 10 & 7.29361 & 1.51762 & 2.59385 \\ 25 & \mathrm{H} 11 & 2.31201 & -2.96726 & 2.21970 \\ 26 & \mathrm{H} 12 & 4.25757 & -4.75229 & 0.78770 \\ 27 & \mathrm{H} 13 & 4.59178 & -4.28664 & 3.83144 \\ 28 & \mathrm{H} 14 & 1.98629 & -8.34352 & -2.19843 \\ 29 & \mathrm{H} 15 & 0.94945 & -5.47693 & -1.86569 \\ 30 & \mathrm{H} 16 & 2.87637 & -6.17956 & -4.14278\end{array}$

Nuclear repulsion energy $=893.233141$

SA-5-CASSCF $(12,11) / 6-31++G(d, p)$ energies:

root number $1 \mathrm{E}=-642.90888336$ a.u. root number $2 \mathrm{E}=\quad-642.82077312$ a.u. root number $3 \mathrm{E}=\quad-642.80633069$ a.u. root number $4 \mathrm{E}=-642.74384509$ a.u. root number $5 \mathrm{E}=\quad-642.73629021$ a.u.

MS-5-CASPT2/6-31++G(d,p) energies:

$\begin{array}{llllll}-644.91779631 & -644.83066879 & -644.79776746 & -644.77436971 & -644.73391454\end{array}$

\section{SA-5-CASSCF $(12,11) / 6-31(+) G(d, p)$ energies:}

root number $1 \mathrm{E}=\quad-642.88966494 \mathrm{a} . u$. root number $2 \mathrm{E}=\quad-642.81466079$ a.u. root number $3 \mathrm{E}=\quad-642.78843683$ a.u. root number $4 \mathrm{E}=\quad-642.73643987$ a.u. root number $5 \mathrm{E}=\quad-642.71788591$ a.u.

\section{MS-5-CASPT2/6-31(+)G(d,p) energies:}

$\begin{array}{llllll}-644.89233434 & -644.80601245 & -644.78114380 & -644.75024403 & -644.71523201\end{array}$

PT2 (optimization led to KETO2; see the manuscript)

Cartesian coordinates:

No. Label X $\quad$ Y $\quad$ Z




\begin{tabular}{|c|c|c|c|c|}
\hline 1 & $\mathrm{C} 1$ & 1.29319 & 1.00143 & -0.93326 \\
\hline 2 & $\mathrm{C} 2$ & -1.04243 & 0.11075 & 0.09515 \\
\hline 3 & C3 & -3.07681 & 1.81772 & 0.17890 \\
\hline 4 & $\mathrm{C} 4$ & -2.68832 & 4.34307 & -0.67447 \\
\hline 5 & $\mathrm{C} 5$ & -0.42399 & 5.26783 & -1.55327 \\
\hline 6 & C6 & 1.72626 & 3.59254 & -1.66983 \\
\hline 7 & $\mathrm{C} 7$ & -5.46085 & 0.88186 & 1.19240 \\
\hline 8 & $\mathrm{C} 8$ & -5.51966 & -1.61089 & 2.03731 \\
\hline 9 & C9 & -3.36365 & -3.14097 & 1.90668 \\
\hline 10 & $\mathrm{O} 1$ & 3.88994 & 4.23829 & -2.32764 \\
\hline 11 & N1 & -1.10208 & -2.29932 & 0.92954 \\
\hline 12 & N2 & 7.18041 & 0.23198 & 1.81508 \\
\hline 13 & N3 & 3.47682 & -4.19539 & 1.96493 \\
\hline 14 & N4 & 2.47456 & -6.39218 & -3.07067 \\
\hline 15 & NX & 3.47682 & -4.19539 & 9 1.96493 \\
\hline 16 & H1 & 2.84032 & -0.28130 & -1.19366 \\
\hline 17 & $\mathrm{H} 2$ & -4.28284 & 5.60984 & -0.58430 \\
\hline 18 & H3 & -0.19839 & 7.19814 & -2.13719 \\
\hline 19 & H4 & -7.09650 & 2.08028 & 1.28934 \\
\hline 20 & H5 & -7.22903 & -2.39824 & 2.81084 \\
\hline 21 & H6 & -3.40289 & -5.06169 & 2.57038 \\
\hline 22 & $\mathrm{H7}$ & 6.52802 & 1.41116 & 0.47520 \\
\hline 23 & H8 & 4.78539 & -2.77688 & 2.01405 \\
\hline 24 & H9 & 8.99930 & -0.11382 & 1.41659 \\
\hline 25 & H10 & 7.14910 & 1.20320 & 3.44139 \\
\hline 26 & H1 1 & 1.57925 & -3.42764 & 1.69690 \\
\hline 27 & H12 & 3.75790 & -5.30205 & 0.42035 \\
\hline 28 & H13 & 3.58199 & -5.22840 & 3.54930 \\
\hline 29 & H14 & 2.01493 & -8.18767 & $7-3.45390$ \\
\hline 30 & H15 & 0.85937 & -5.41483 & $3-2.86990$ \\
\hline 31 & H16 & 3.36599 & -5.70274 & -4.59212 \\
\hline
\end{tabular}

Nuclear repulsion energy $=885.146156$

SA-5-CASSCF $(12,11) / 6-31++G(d, p)$ energies:

root number $1 \mathrm{E}=\quad-642.91300561$ a.u.

root number $2 \mathrm{E}=-642.83224646$ a.u.

root number $3 \mathrm{E}=-642.83069752$ a.u.

root number $4 \mathrm{E}=-642.75083117$ a.u.

root number $5 \mathrm{E}=\quad-642.74935163$ a.u.

MS-5-CASPT2/6-31++G(d,p) energies:

$\begin{array}{llllll}-644.91518233 & -644.84158822 & -644.79966535 & -644.77866719 & -644.73402119\end{array}$

\section{SA-5-CASSCF $(12,11) / 6-31(+) G(d, p)$ energies:}

root number $1 \mathrm{E}=\quad-642.89224348$ a.u.

root number $2 \mathrm{E}=\quad-642.82308152$ a.u.

root number $3 \mathrm{E}=\quad-642.81148729$ a.u.

root number $4 \mathrm{E}=\quad-642.73950370$ a.u. 
root number $5 \mathrm{E}=\quad-642.73083168$ a.u.

MS-5-CASPT2/6-31(+)G(d,p) energies:

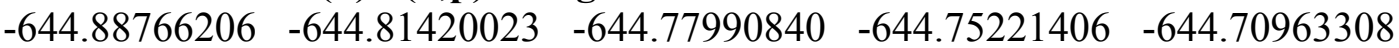

\section{KETO2}

Cartesian coordinates:

\begin{tabular}{|c|c|c|c|c|}
\hline \multicolumn{2}{|c|}{ No. Label } & $\mathrm{X}$ & Y $\quad Z$ & \\
\hline 1 & $\mathrm{C} 1$ & -1.29273 & -1.00931 & -0.81417 \\
\hline 2 & $\mathrm{C} 2$ & -3.29538 & -2.67109 & -0.53720 \\
\hline 3 & $\mathrm{C} 3$ & -5.84228 & -1.69135 & -1.05931 \\
\hline 4 & $\mathrm{C} 4$ & -6.10546 & 0.78712 & -1.78370 \\
\hline 5 & $\mathrm{C} 5$ & -3.97169 & 2.38292 & -2.02523 \\
\hline 6 & C6 & -2.72708 & -5.19445 & 0.23038 \\
\hline 7 & $\mathrm{C} 7$ & -0.32993 & -6.05032 & 0.70296 \\
\hline 8 & $\mathrm{C} 8$ & 1.82486 & -4.37200 & 0.43364 \\
\hline 9 & C9 & 1.27169 & -1.81586 & -0.34241 \\
\hline 10 & $\mathrm{O} 1$ & 4.04237 & -5.01518 & 0.82734 \\
\hline & $\mathrm{N} 1$ & -1.62196 & 1.41929 & -1.52838 \\
\hline 12 & $\mathrm{~N} 2$ & 7.74169 & -0.01046 & -0.40443 \\
\hline 13 & N3 & 3.12954 & 4.59389 & -1.80478 \\
\hline 14 & N4 & 2.82496 & 8.76800 & 2.92636 \\
\hline 15 & H1 & 2.80546 & -0.51504 & -0.57055 \\
\hline 16 & $\mathrm{H} 2$ & -4.28459 & -6.48909 & 0.44595 \\
\hline 17 & H3 & 0.02144 & -7.96491 & 1.27794 \\
\hline 18 & $\mathrm{H} 4$ & -7.45790 & -2.90671 & -0.87640 \\
\hline 19 & H5 & -7.93740 & 1.56829 & -2.18365 \\
\hline 20 & H6 & -4.06458 & 4.32276 & -2.58610 \\
\hline 21 & $\mathrm{H} 7$ & 6.94876 & -1.67017 & 0.06445 \\
\hline 22 & $\mathrm{H} 8$ & 4.61557 & 3.46648 & -1.43830 \\
\hline 23 & H9 & 8.86528 & 0.50180 & 1.03016 \\
\hline 24 & $\mathrm{H} 10$ & 8.85917 & $7-0.32731$ & $\begin{array}{ll}1 & -1.89919\end{array}$ \\
\hline 25 & H11 & -0.08260 & 2.54476 & $6-1.68592$ \\
\hline & H12 & 3.09267 & 7.95180 & $0 \quad-0.47494$ \\
\hline 27 & H13 & 3.46355 & 5.43425 & $5-3.46842$ \\
\hline 28 & H14 & 2.40102 & 10.58394 & 42.60696 \\
\hline 29 & H15 & 1.43454 & 8.03313 & $\begin{array}{ll}3 & 3.97857\end{array}$ \\
\hline 30 & H16 & 4.40655 & 8.72428 & $8 \quad 3.96380$ \\
\hline
\end{tabular}

Nuclear repulsion energy $=850.369133$

\section{SA-5-CASSCF $(12,11) / 6-31++G(d, p)$ energies:}

root number $1 \mathrm{E}=\quad-642.93561630$ a.u.

root number $2 \mathrm{E}=\quad-642.86998465$ a.u.

root number $3 \mathrm{E}=-642.81521189$ a.u.

root number $4 \mathrm{E}=\quad-642.79244527$ a.u. 
root number $5 \mathrm{E}=-642.75983924$ a.u.

MS-5-CASPT2/6-31++G(d,p) energies:

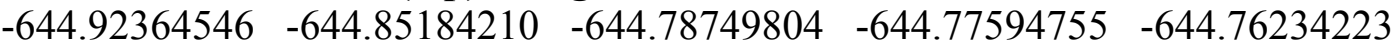

\section{SA-5-CASSCF(12,11)/6-31(+)G(d,p) energies: \\ root number $1 \mathrm{E}=\quad-642.91437153$ a.u. \\ root number $2 \mathrm{E}=-642.84915539$ a.u. \\ root number $3 \mathrm{E}=-642.80161952$ a.u. \\ root number $4 \mathrm{E}=\quad-642.77179820$ a.u. \\ root number $5 \mathrm{E}=-642.73863387$ a.u.}

\section{MS-5-CASPT2/6-31(+)G(d,p) energies:}

$\begin{array}{llllll}-644.89647059 & -644.82523854 & -644.76109004 & -644.75404186 & -644.73705004\end{array}$

****************************************************************************

************************************************************************

\section{$\mathrm{C}_{\mathrm{S}}$-symmetry pathway}

\section{Levels of calculation}

Geometries (S1 state): SA-3-CASSCF(12,12)/6-31(+)G(d,p)

Energies: SA-5-CASSCF $(12,12)$ and MS-5-CASPT2 $(12,12)$ with the 6-31 $(+) \mathrm{G}(\mathrm{d}, \mathrm{p})$

basis sets

Cartesian coordinates are given in Bohr and energies in a.u.

\section{ENOL}

Cartesian coordinates:

\begin{tabular}{|c|c|c|c|}
\hline No. Label & $\mathrm{X}$ & $\mathrm{Y}$ & \\
\hline $1 \mathrm{C} 1$ & -0.00722 & 0.00000 & -0.15351 \\
\hline $2 \mathrm{C} 2$ & -0.02982 & 0.00000 & 2.50477 \\
\hline 3 C3 & 2.22292 & 0.00000 & 3.92518 \\
\hline $4 \quad \mathrm{C} 4$ & 4.69403 & 0.00000 & 2.56534 \\
\hline $5 \quad \mathrm{C} 5$ & 4.66162 & 0.00000 & -0.08714 \\
\hline 6 C6 & 2.35176 & 0.00000 & -1.44085 \\
\hline $7 \quad \mathrm{C} 7$ & 2.30727 & 0.00000 & 6.60575 \\
\hline $8 \mathrm{C} 8$ & 6.91468 & 0.00000 & 6.33397 \\
\hline 9 C9 & 4.67302 & 0.00000 & 7.80867 \\
\hline $10 \mathrm{O}$ & 2.27908 & 0.00000 & -3.95186 \\
\hline $11 \mathrm{~N} 1$ & 6.77572 & 0.00000 & -6.86160 \\
\hline $12 \mathrm{~N} 2$ & 12.45608 & 0.00000 & $\begin{array}{ll}0 & -4.74991\end{array}$ \\
\hline $13 \mathrm{~N} 3$ & 12.70838 & 0.00000 & ) 1.39766 \\
\hline 14 N4 & 6.92236 & 0.00000 & 3.78378 \\
\hline
\end{tabular}




$\begin{array}{llccc}15 & \text { H1 } & 0.58631 & 0.00000 & 7.68426 \\ 16 & \text { H2 } & -1.71914 & 0.00000 & -1.23826 \\ 17 & \text { H3 } & -1.80615 & 0.00000 & 3.49356 \\ 18 & \text { H4 } & 6.42702 & 0.00000 & -1.08862 \\ 19 & \text { H5 } & 8.73387 & 0.00000 & 7.23583 \\ 20 & \text { H6 } & 4.81300 & 0.00000 & 9.83389 \\ 21 & \text { H7 } & 6.64471 & 1.52052 & -7.98362 \\ 22 & \text { H8 } & 8.52671 & 0.00000 & -6.10528 \\ 23 & \text { H9 } & 13.41935 & 1.51998 & -5.33916 \\ 24 & \text { H10 } & 12.50945 & 0.00000 & -2.84664 \\ 25 & \text { H11 } & 13.54889 & 1.52328 & 2.14317 \\ 26 & \text { H12 } & 10.90196 & 0.00000 & 1.98496 \\ 27 & \text { H13 } & 3.92285 & 0.00000 & -4.74311 \\ 28 & \text { H7 } & 6.64471 & -1.52052 & -7.98362 \\ 29 & \text { H9 } & 13.41935 & -1.51998 & -5.33916 \\ 30 & \text { H11 } & 13.54889 & -1.52328 & 2.14317\end{array}$

Nuclear repulsion energy $=846.765701$

SA-5-CASSCF(12,11)/6-31(+)G(d,p) energies for symmetry species 1:

root number $1 \mathrm{E}=\quad-642.97064398$ a.u.

root number $2 \mathrm{E}=-642.83359872$ a.u.

root number $3 \mathrm{E}=\quad-642.77482039$ a.u.

root number $4 \mathrm{E}=-642.75848123$ a.u.

root number $5 \mathrm{E}=-642.74379844$ a.u.

MS-5-CASPT2/6-31(+)G(d,p) energies for symmetry species 1:

$\begin{array}{llllll}-644.92497381 & -644.80155965 & -644.76832276 & -644.71966699 & -644.71554331\end{array}$

\section{SA-5-CASSCF(12,11)/6-31(+)G(d,p) energies for symmetry species 2:}

root number $1 \mathrm{E}=-642.77659636$ a.u.

root number $2 \mathrm{E}=-642.73609573$ a.u.

root number $3 \mathrm{E}=-642.67693534$ a.u.

root number $4 \mathrm{E}=-642.65920038$ a.u.

root number $5 \mathrm{E}=\quad-642.61874812$ a.u.

MS-5-CASPT2/6-31(+)G(d,p) energies for symmetry species 2:

$\begin{array}{llllll}-644.73085821 & -644.69894078 & -644.64063638 & -644.62352476 & -644.58928742\end{array}$

\section{MECP1}

Cartesian coordinates:

\begin{tabular}{ccccc} 
No. & Label & X & \multicolumn{2}{c}{ Y } \\
- \hdashline 1 & C1 & 1.94248 & 0.00000 & -0.46056 \\
2 & C2 & 4.11456 & 0.00000 & 1.16925 \\
3 & C3 & 6.53741 & 0.00000 & 0.01599 \\
4 & C4 & 6.66402 & 0.00000 & -2.62248
\end{tabular}




$\begin{array}{clccc}5 & \mathrm{C} 5 & 4.41744 & 0.00000 & -4.04652 \\ 6 & \mathrm{C} 6 & -0.46063 & 0.00000 & 0.66030 \\ 7 & \mathrm{C} 7 & -0.81078 & 0.00000 & 3.35664 \\ 8 & \mathrm{C} 8 & 1.40597 & 0.00000 & 4.95337 \\ 9 & \mathrm{C} 9 & 3.75287 & 0.00000 & 3.85723 \\ 10 & \mathrm{O} & -2.97209 & 0.00000 & 4.31420 \\ 11 & \mathrm{~N} 1 & 2.10217 & 0.00000 & -3.00940 \\ 12 & \mathrm{~N} 2 & -7.87882 & 0.00000 & 2.63107 \\ 13 & \mathrm{~N} 3 & -8.45490 & 0.00000 & -2.96346 \\ 14 & \mathrm{~N} 4 & -3.14516 & 0.00000 & -6.20638 \\ 15 & \mathrm{H} 1 & 8.21999 & 0.00000 & 1.15176 \\ 16 & \mathrm{H} 2 & 1.13354 & 0.00000 & 6.96194 \\ 17 & \mathrm{H} 3 & 5.41128 & 0.00000 & 5.03400 \\ 18 & \mathrm{H} 4 & -2.10310 & 0.00000 & -0.52930 \\ 19 & \mathrm{H} 5 & 4.50020 & 0.00000 & -6.07654 \\ 20 & \mathrm{H} 6 & 8.45455 & 0.00000 & -3.57972 \\ 21 & \mathrm{H} 7 & -8.79731 & 1.53270 & 3.29420 \\ 22 & \mathrm{H} 8 & -7.95467 & 0.00000 & 0.68737 \\ 23 & \mathrm{H} 9 & -9.45583 & 1.51793 & -3.49862 \\ 24 & \mathrm{H} 10 & -6.84236 & 0.00000 & -3.97845 \\ 25 & \mathrm{H} 11 & -3.21167 & 1.51312 & -7.33555 \\ 26 & \mathrm{H} 12 & -1.43575 & 0.00000 & -5.38556 \\ 27 & \mathrm{H} 13 & -5.90994 & 0.00000 & 3.36802 \\ 28 & \mathrm{H} 7 & -8.79731 & -1.53270 & 3.29420 \\ 29 & \mathrm{H} 9 & -9.45583 & -1.51793 & -3.49862 \\ 30 & \mathrm{H} 11 & -3.21167 & -1.51312 & -7.33555\end{array}$

Nuclear repulsion energy $=852.015465$

\section{SA-5-CASSCF $(12,11) / 6-31(+) G(d, p)$ energies for symmetry species 1:}

root number $1 \mathrm{E}=\quad-642.90910888$ a.u.

root number $2 \mathrm{E}=-642.80315169$ a.u.

root number $3 \mathrm{E}=\quad-642.78326331$ a.u.

root number $4 \mathrm{E}=-642.73024361$ a.u.

root number $5 \mathrm{E}=\quad-642.72181842$ a.u.

MS-5-CASPT2/6-31(+)G(d,p) energies for symmetry species 1:

$\begin{array}{llllll}-644.89651851 & -644.79490082 & -644.75479678 & -644.74370593 & -644.68754271\end{array}$

\section{SA-5-CASSCF $(12,11) / 6-31(+) G(d, p)$ energies for symmetry species 2:}

root number $1 \mathrm{E}=\quad-642.84776688$ a.u.

root number $2 \mathrm{E}=-642.76456274$ a.u.

root number $3 \mathrm{E}=-642.73644044$ a.u.

root number $4 \mathrm{E}=\quad-642.70747401$ a.u.

root number $5 \mathrm{E}=-642.68399842$ a.u.

MS-5-CASPT2/6-31(+)G(d,p) energies for symmetry species 2:

$\begin{array}{llllll}-644.79042406 & -644.72251306 & -644.68382693 & -644.66276579 & -644.64299370\end{array}$ 


\section{HT1}

Cartesian coordinates:

\begin{tabular}{|c|c|c|c|c|}
\hline \multicolumn{2}{|c|}{ No. Label } & $1 \quad X$ & Y $\quad Z$ & \\
\hline 1 & $\mathrm{C} 1$ & 4.52733 & 0.00000 & 2.92912 \\
\hline 2 & $\mathrm{C} 2$ & 2.18038 & 0.00000 & 4.24659 \\
\hline 3 & $\mathrm{C} 3$ & 2.24270 & 0.00000 & 6.90273 \\
\hline 4 & $\mathrm{C} 4$ & 4.55585 & 0.00000 & 8.11793 \\
\hline 5 & $\mathrm{C} 5$ & 6.78988 & 0.00000 & 6.64027 \\
\hline 6 & C6 & 4.54980 & 0.00000 & 0.27337 \\
\hline 7 & $\mathrm{C} 7$ & 2.22032 & $0.00000-$ & -1.16001 \\
\hline 8 & $\mathrm{C} 8$ & -0.15923 & 0.00000 & 0.25431 \\
\hline 9 & C9 & -0.15388 & 0.00000 & 2.81725 \\
\hline 10 & $\mathrm{O}$ & 2.23704 & $0.00000-$ & -3.48948 \\
\hline 11 & N1 & 6.79575 & 0.00000 & 4.16370 \\
\hline 12 & $\mathrm{~N} 2$ & 7.24341 & 0.00000 & -7.22204 \\
\hline 13 & N3 & 12.28450 & 0.00000 & 0.75004 \\
\hline 14 & H1 & 0.50854 & 0.00000 & 7.96233 \\
\hline 15 & $\mathrm{H} 2$ & -1.88057 & 0.00000 & -0.82099 \\
\hline 16 & H3 & -1.90693 & 0.00000 & 3.84620 \\
\hline 17 & H4 & 6.31198 & 0.00000 & -0.72805 \\
\hline 18 & H5 & 8.60629 & 0.00000 & 7.55426 \\
\hline 19 & H6 & 4.68786 & 0.00000 & 10.14288 \\
\hline 20 & $\mathrm{H} 7$ & 7.15010 & 1.52096 & -8.34939 \\
\hline 21 & H8 & 10.51498 & 0.00000 & -5.63581 \\
\hline 22 & H9 & 13.27020 & 1.53495 & -5.44836 \\
\hline 23 & H10 & 12.21014 & 0.00000 & $0 \quad-2.90729$ \\
\hline 24 & H11 & 13.29704 & 1.52206 & $5 \quad 1.25097$ \\
\hline 25 & H12 & 10.69026 & 0.00000 & 1.78162 \\
\hline 26 & H13 & 5.68590 & 0.00000 & -6.13787 \\
\hline 27 & N4 & 12.29616 & 0.00000 & -4.84986 \\
\hline 28 & $\mathrm{H} 7$ & 7.15010 & -1.52096 & -8.34939 \\
\hline 29 & H9 & 13.27020 & -1.53495 & -5.44836 \\
\hline 30 & H11 & 13.29704 & -1.52206 & $\begin{array}{ll}6 & 1.25097\end{array}$ \\
\hline
\end{tabular}

Nuclear repulsion energy $=844.445102$

SA-5-CASSCF $(12,11) / 6-31(+) G(d, p)$ energies for symmetry species 1:

root number $1 \mathrm{E}=-642.87882370$ a.u.

root number $2 \mathrm{E}=-642.82903148$ a.u.

root number $3 \mathrm{E}=\quad-642.76113202$ a.u.

root number $4 \mathrm{E}=-642.73866193$ a.u.

root number $5 \mathrm{E}=-642.71728923$ a.u.

MS-5-CASPT2/6-31(+)G(d,p) energies for symmetry species 1:

$\begin{array}{llllll}-644.86131466 & -644.76511972 & -644.76157271 & -644.68529949 & -644.65501993\end{array}$

SA-5-CASSCF(12,11)/6-31(+)G(d,p) energies for symmetry species 2: 
root number $1 \mathrm{E}=\quad-642.86791654$ a.u.

root number $2 \mathrm{E}=\quad-642.77679548$ a.u.

root number $3 \mathrm{E}=-642.75696826$ a.u.

root number $4 \mathrm{E}=-642.72238995$ a.u.

root number $5 \mathrm{E}=-642.69815783$ a.u.

MS-5-CASPT2/6-31(+)G(d,p) energies for symmetry species 2:

$\begin{array}{llllll}-644.79972530 & -644.72354949 & -644.69262757 & -644.66688966 & -644.64482594\end{array}$

$\mathbf{T S}_{1 / 2}$

Cartesian coordinates:

\begin{tabular}{clcccc}
\hline No. & Label & X & Y & Z \\
----------- & \\
1 & C1 & 4.72492 & 0.00000 & 3.17532 \\
2 & C2 & 2.27650 & 0.00000 & 4.29275 \\
3 & C3 & 2.11849 & 0.00000 & 6.94452 \\
4 & C4 & 4.32339 & 0.00000 & 8.34689 \\
5 & C5 & 6.67256 & 0.00000 & 7.06022 \\
6 & C6 & 0.06760 & 0.00000 & 2.67206 \\
7 & C7 & 0.27468 & 0.00000 & 0.11884 \\
8 & C8 & 2.76607 & 0.00000 & -1.08917 \\
9 & C9 & 4.96966 & 0.00000 & 0.53090 \\
10 & O & 2.98217 & 0.00000 & -3.40825 \\
11 & N1 & 6.88419 & 0.00000 & 4.59203 \\
12 & N2 & 7.37237 & 0.00000 & -7.40092 \\
13 & N3 & 11.66477 & 0.00000 & -5.09206 \\
14 & N4 & 12.05497 & 0.00000 & 0.73414 \\
15 & H1 & 0.30255 & 0.00000 & 7.85697 \\
16 & H2 & -1.34986 & 0.00000 & -1.09750 \\
17 & H3 & -1.76488 & 0.00000 & 3.55174 \\
18 & H4 & 6.80990 & 0.00000 & -0.31750 \\
19 & H5 & 8.40673 & 0.00000 & 8.12198 \\
20 & H6 & 4.28645 & 0.00000 & 10.37588 \\
21 & H7 & 7.31848 & 1.53078 & -8.53310 \\
22 & H8 & 12.62843 & 1.52435 & -5.70059 \\
23 & H9 & 11.64273 & 0.00000 & -3.17148 \\
24 & H10 & 13.15404 & 1.51698 & 1.02047 \\
25 & H11 & 10.72003 & 0.00000 & 2.08248 \\
26 & H12 & 5.83467 & 0.00000 & -6.27362 \\
27 & H13 & 9.41841 & 0.00000 & -6.20016 \\
28 & H7 & 7.31848 & -1.53078 & -8.53310 \\
29 & H8 & 12.62843 & -1.52435 & -5.70059 \\
30 & H10 & 13.15404 & -1.51698 & 1.02047
\end{tabular}

Nuclear repulsion energy $=850.933053$

SA-5-CASSCF $(12,11) / 6-31(+) G(d, p)$ energies for symmetry species 1: 
root number $1 \mathrm{E}=-642.87860844$ a.u.

root number $2 \mathrm{E}=\quad-642.81592055$ a.u.

root number $3 \mathrm{E}=-642.75581050$ a.u.

root number $4 \mathrm{E}=-642.72560756$ a.u.

root number $5 \mathrm{E}=-642.70395166$ a.u.

MS-5-CASPT2/6-31(+)G(d,p) energies for symmetry species 1:

$-644.87057998 \quad-644.77074118 \quad-644.75859970 \quad-644.68287551 \quad-644.65330730$

SA-5-CASSCF $(12,11) / 6-31(+) G(d, p)$ energies for symmetry species 2:

root number $1 \mathrm{E}=-642.85323232$ a.u.

root number $2 \mathrm{E}=-642.76235453$ a.u.

root number $3 \mathrm{E}=-642.74212213$ a.u.

root number $4 \mathrm{E}=-642.70778963$ a.u.

root number $5 \mathrm{E}=-642.68274176$ a.u.

MS-5-CASPT2/6-31(+)G(d,p) energies for symmetry species 2:

$\begin{array}{llllll}-644.79619152 & -644.72032514 & -644.68902034 & -644.66358191 & -644.64090166\end{array}$

\section{HT2}

Cartesian coordinates:

\begin{tabular}{|c|c|c|c|c|}
\hline \multicolumn{2}{|c|}{ No. Label } & $X$ & \multicolumn{2}{|l|}{ Y $\quad Z$} \\
\hline 1 & $\mathrm{C} 1$ & 4.52733 & 0.00000 & 2.92912 \\
\hline 2 & $\mathrm{C} 2$ & 2.18038 & 0.00000 & 4.24659 \\
\hline 3 & $\mathrm{C} 3$ & 2.24270 & 0.00000 & 6.90273 \\
\hline 4 & $\mathrm{C} 4$ & 4.55585 & 0.00000 & 8.11793 \\
\hline 5 & $\mathrm{C} 5$ & 6.78988 & 0.00000 & 6.64027 \\
\hline 6 & C6 & 4.54980 & 0.00000 & 0.27337 \\
\hline 7 & $\mathrm{C} 7$ & 2.22032 & $0.00000-$ & -1.16001 \\
\hline 8 & $\mathrm{C} 8$ & -0.15923 & 0.00000 & 0.25431 \\
\hline 9 & C9 & -0.15388 & 0.00000 & 2.81725 \\
\hline 10 & $\mathrm{O}$ & 2.23704 & $0.00000-$ & -3.48948 \\
\hline 11 & N1 & 6.79575 & 0.00000 & 4.16370 \\
\hline 12 & N2 & 7.24341 & 0.00000 & -7.22204 \\
\hline 13 & N3 & 12.28450 & 0.00000 & 0.75004 \\
\hline 14 & H1 & 0.50854 & 0.00000 & 7.96233 \\
\hline 15 & $\mathrm{H} 2$ & -1.88057 & 0.00000 & -0.82099 \\
\hline 16 & H3 & -1.90693 & 0.00000 & 3.84620 \\
\hline 17 & $\mathrm{H} 4$ & 6.31198 & 0.00000 & -0.72805 \\
\hline 18 & H5 & 8.60629 & 0.00000 & 7.55426 \\
\hline 19 & H6 & 4.68786 & 0.00000 & 10.14288 \\
\hline 20 & H7 & 7.15010 & 1.52096 & -8.34939 \\
\hline 21 & $\mathrm{H} 8$ & 10.51498 & 0.00000 & -5.63581 \\
\hline 22 & H9 & 13.27020 & 1.53495 & -5.44836 \\
\hline 23 & H10 & 12.21014 & 0.00000 & -2.90729 \\
\hline 24 & H11 & 13.29704 & 1.52206 & $5 \quad 1.25097$ \\
\hline
\end{tabular}


$\begin{array}{lllll}25 & \mathrm{H} 12 & 10.69026 & 0.00000 & 1.78162\end{array}$

$\begin{array}{lllll}26 & \mathrm{H} 13 & 5.68590 & 0.00000 & -6.13787\end{array}$

$\begin{array}{llllll}27 & \mathrm{~N} 4 & 12.29616 & 0.00000 & -4.84986\end{array}$

28 H7 $\quad 7.15010-1.52096-8.34939$

$\begin{array}{lllll}29 & \text { H9 } & 13.27020 & -1.53495 & -5.44836\end{array}$

$\begin{array}{lllll}30 & \mathrm{H} 11 & 13.29704 & -1.52206 & 1.25097\end{array}$

Nuclear repulsion energy $=844.445102$

\section{SA-5-CASSCF $(12,11) / 6-31(+) G(d, p)$ energies for symmetry species 1:}

root number $1 \mathrm{E}=-642.87882370$ a.u.

root number $2 \mathrm{E}=\quad-642.82903148$ a.u.

root number $3 \mathrm{E}=\quad-642.76113202$ a.u.

root number $4 \mathrm{E}=-642.73866193$ a.u.

root number $5 \mathrm{E}=-642.71728923$ a.u.

MS-5-CASPT2/6-31(+)G(d,p) energies for symmetry species 1:

$\begin{array}{llllll}-644.86131466 & -644.76511972 & -644.76157271 & -644.68529949 & -644.65501993\end{array}$

\section{SA-5-CASSCF $(12,11) / 6-31(+) G(d, p)$ energies for symmetry species 2:}

root number $1 \mathrm{E}=\quad-642.86791654$ a.u.

root number $2 \mathrm{E}=-642.77679548$ a.u.

root number $3 \mathrm{E}=\quad-642.75696826$ a.u.

root number $4 \mathrm{E}=-642.72238995$ a.u.

root number $5 \mathrm{E}=-642.69815783$ a.u.

MS-5-CASPT2/6-31(+)G(d,p) energies for symmetry species 2:

$\begin{array}{llllll}-644.79972530 & -644.72354949 & -644.69262757 & -644.66688966 & -644.64482594\end{array}$

$\mathbf{T S}_{2 / 3}$

Cartesian coordinates:

\begin{tabular}{|c|c|c|c|}
\hline No. Label & $\mathrm{X}$ & Y & \\
\hline $1 \mathrm{C} 1$ & 4.49517 & 0.00000 & 2.62232 \\
\hline $2 \mathrm{C} 2$ & 2.22780 & 0.00000 & 4.07210 \\
\hline 3 C3 & 2.44069 & 0.00000 & 6.72075 \\
\hline $4 \mathrm{C} 4$ & 4.81793 & 0.00000 & 7.80322 \\
\hline $5 \quad \mathrm{C} 5$ & 6.96559 & 0.00000 & 6.20232 \\
\hline 6 C6 & -0.18474 & 0.00000 & 2.77936 \\
\hline $7 \quad$ C7 & -0.33858 & 0.00000 & 0.22101 \\
\hline $8 \mathrm{C} 8$ & 1.95580 & 0.00000 & -1.32687 \\
\hline 9 C9 & 4.36380 & 0.00000 & -0.02935 \\
\hline $10 \mathrm{O}$ & 1.84286 & 0.00000 & -3.65390 \\
\hline $11 \mathrm{~N} 1$ & 6.83027 & 0.00000 & 3.72922 \\
\hline $12 \mathrm{~N} 2$ & 12.32371 & 0.00000 & 0.80860 \\
\hline $13 \mathrm{~N} 3$ & 12.31225 & 0.00000 & ] -4.05897 \\
\hline $14 \quad \mathrm{~N} 4$ & 7.15437 & 0.00000 & -7.02026 \\
\hline
\end{tabular}




$\begin{array}{llccc}15 & \text { H1 } & 0.76908 & 0.00000 & 7.87643 \\ 16 & \text { H2 } & -2.11914 & 0.00000 & -0.75309 \\ 17 & \text { H3 } & -1.87509 & 0.00000 & 3.90831 \\ 18 & \text { H4 } & 6.05938 & 0.00000 & -1.13933 \\ 19 & \text { H5 } & 8.83001 & 0.00000 & 7.01278 \\ 20 & \text { H6 } & 5.06563 & 0.00000 & 9.81708 \\ 21 & \text { H7 } & 7.18011 & 1.52134 & -8.14841 \\ 22 & \text { H8 } & 10.60409 & 0.00000 & -4.93326 \\ 23 & \text { H9 } & 13.29700 & 1.52644 & -4.62686 \\ 24 & \text { H10 } & 13.30626 & 1.52914 & 1.38036 \\ 25 & \text { H11 } & 10.61691 & 0.00000 & 1.66519 \\ 26 & \text { H12 } & 5.48505 & 0.00000 & -6.12056 \\ 27 & \text { H13 } & 12.22765 & 0.00000 & -1.60049 \\ 28 & \text { H7 } & 7.18011 & -1.52134 & -8.14841 \\ 29 & \text { H9 } & 13.29700 & -1.52644 & -4.62686 \\ 30 & \text { H10 } & 13.30626 & -1.52914 & 1.38036\end{array}$

Nuclear repulsion energy $=851.575636$

SA-5-CASSCF(12,11)/6-31(+)G(d,p) energies for symmetry species 1:

root number $1 \mathrm{E}=-642.87861756$ a.u.

root number $2 \mathrm{E}=-642.81166890$ a.u.

root number $3 \mathrm{E}=\quad-642.76683729$ a.u.

root number $4 \mathrm{E}=-642.72151203$ a.u.

root number $5 \mathrm{E}=-642.70329668$ a.u.

MS-5-CASPT2/6-31(+)G(d,p) energies for symmetry species 1:

$\begin{array}{llllll}-644.86865321 & -644.77508345 & -644.76042287 & -644.72496107 & -644.68372375\end{array}$

SA-5-CASSCF(12,11)/6-31(+)G(d,p) energies for symmetry species 2:

root number $1 \mathrm{E}=-642.85458078$ a.u.

root number $2 \mathrm{E}=-642.76339128$ a.u.

root number $3 \mathrm{E}=-642.74377723$ a.u.

root number $4 \mathrm{E}=-642.70900221$ a.u.

root number $5 \mathrm{E}=-642.68481983$ a.u.

MS-5-CASPT2/6-31(+)G(d,p) energies for symmetry species 2:

$\begin{array}{llllll}-644.79750503 & -644.72135610 & -644.69058295 & -644.66461489 & -644.64261735\end{array}$

\section{MECP2}

Cartesian coordinates:

\begin{tabular}{|c|c|c|c|}
\hline No. Label & $X$ & Y & \\
\hline $1 \mathrm{C} 1$ & -0.63451 & 0.00000 & 1.66365 \\
\hline $2 \mathrm{C} 2$ & -2.90249 & 0.00000 & 3.07927 \\
\hline $3 \mathrm{C} 3$ & -2.71663 & 0.00000 & 5.76029 \\
\hline $4 \mathrm{C} 4$ & -0.35530 & 0.00000 & 6.86460 \\
\hline
\end{tabular}




$\begin{array}{clccc}5 & \mathrm{C} 5 & 1.81988 & 0.00000 & 5.30506 \\ 6 & \mathrm{C} 6 & -5.28329 & 0.00000 & 1.75371 \\ 7 & \mathrm{C} 7 & -5.41817 & 0.00000 & -0.81117 \\ 8 & \mathrm{C} 8 & -3.11694 & 0.00000 & -2.33637 \\ 9 & \mathrm{C} 9 & -0.72478 & 0.00000 & -1.00857 \\ 10 & \mathrm{O} & -3.19361 & 0.00000 & -4.66623 \\ 11 & \mathrm{~N} 1 & 7.01146 & 0.00000 & 0.35712 \\ 12 & \mathrm{~N} 2 & 6.98481 & 0.00000 & -5.25471 \\ 13 & \mathrm{~N} 3 & 1.72746 & 0.00000 & -8.38907 \\ 14 & \mathrm{~N} 4 & 1.67929 & 0.00000 & 2.80319 \\ 15 & \mathrm{H} 1 & -4.40102 & 0.00000 & 6.89574 \\ 16 & \mathrm{H} 2 & -7.19239 & 0.00000 & -1.79640 \\ 17 & \mathrm{H} 3 & -6.98955 & 0.00000 & 2.85910 \\ 18 & \mathrm{H} 4 & 0.98478 & 0.00000 & -2.09957 \\ 19 & \mathrm{H} 5 & 3.68077 & 0.00000 & 6.11456 \\ 20 & \mathrm{H} 6 & -0.12897 & 0.00000 & 8.88102 \\ 21 & \mathrm{H} 7 & 1.65297 & 1.52146 & -9.50933 \\ 22 & \mathrm{H} 8 & 5.31196 & 0.00000 & -6.16905 \\ 23 & \mathrm{H} 9 & 7.95487 & 1.51924 & -5.84063 \\ 24 & \mathrm{H} 10 & 6.94878 & 0.00000 & -1.59023 \\ 25 & \mathrm{H} 11 & 7.98469 & 1.53183 & 0.95339 \\ 26 & \mathrm{H} 12 & 0.15495 & 0.00000 & -7.34117 \\ 27 & \mathrm{H} 13 & 4.81343 & 0.00000 & 1.29026 \\ 28 & \mathrm{H} 7 & 1.65297 & -1.52146 & -9.50933 \\ 29 & \mathrm{H} 9 & 7.95487 & -1.51924 & -5.84063 \\ 30 & \mathrm{H} 11 & 7.98469 & -1.53183 & 0.95339 \\ & & & & \end{array}$

Nuclear repulsion energy $=849.016996$

\section{SA-5-CASSCF $(12,11) / 6-31(+) G(d, p)$ energies for symmetry species 1:}

root number $1 \mathrm{E}=\quad-642.87409566$ a.u.

root number $2 \mathrm{E}=-642.78921957$ a.u.

root number $3 \mathrm{E}=-642.77660593$ a.u.

root number $4 \mathrm{E}=-642.70428438$ a.u.

root number $5 \mathrm{E}=-642.70088319$ a.u.

MS-5-CASPT2/6-31(+)G(d,p) energies for symmetry species 1:

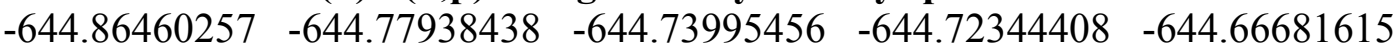

\section{SA-5-CASSCF $(12,11) / 6-31(+) G(d, p)$ energies for symmetry species 2:}

root number $1 \mathrm{E}=\quad-642.83548100$ a.u.

root number $2 \mathrm{E}=-642.74559192$ a.u.

root number $3 \mathrm{E}=-642.72244498$ a.u.

root number $4 \mathrm{E}=\quad-642.69062123$ a.u.

root number $5 \mathrm{E}=-642.66800964$ a.u.

MS-5-CASPT2/6-31(+)G(d,p) energies for symmetry species 2:

$\begin{array}{llllll}-644.77917458 & -644.70469495 & -644.67057738 & -644.64710825 & -644.62773607\end{array}$ 


\section{KETO}

Cartesian coordinates:

\begin{tabular}{clcccc}
\hline No. & Label & X & Y & Z \\
\hline------------ \\
1 & C1 & 4.65869 & 0.00000 & 2.76782 \\
2 & C2 & 2.39839 & 0.00000 & 4.09383 \\
3 & C3 & 2.53594 & 0.00000 & 6.86725 \\
4 & C4 & 4.87041 & 0.00000 & 8.00503 \\
5 & C5 & 7.10562 & 0.00000 & 6.54052 \\
6 & C6 & 0.10656 & 0.00000 & 2.67516 \\
7 & C7 & 0.01829 & 0.00000 & 0.08957 \\
8 & C8 & 2.33548 & 0.00000 & -1.37913 \\
9 & C9 & 4.67468 & 0.00000 & 0.03571 \\
10 & O & 2.37715 & 0.00000 & -3.71927 \\
11 & N1 & 12.02085 & 0.00000 & 1.45902 \\
12 & N2 & 12.24817 & 0.00000 & -4.58916 \\
13 & N3 & 6.93391 & 0.00000 & -7.65160 \\
14 & H1 & 0.83099 & 0.00000 & 7.96805 \\
15 & H2 & -1.74272 & 0.00000 & -0.91870 \\
16 & H3 & -1.64534 & 0.00000 & 3.71323 \\
17 & H4 & 6.43130 & 0.00000 & -0.98257 \\
18 & H5 & 8.97159 & 0.00000 & 7.31660 \\
19 & H6 & 5.04761 & 0.00000 & 10.02830 \\
20 & H7 & 6.69758 & 1.52032 & -8.75493 \\
21 & H8 & 10.56796 & 0.00000 & -5.48722 \\
22 & H9 & 13.20286 & 1.51919 & -5.19533 \\
23 & H10 & 11.98893 & 0.00000 & -0.44908 \\
24 & H11 & 13.01398 & 1.52035 & 1.99828 \\
25 & H12 & 5.51502 & 0.00000 & -6.39087 \\
26 & H13 & 8.53976 & 0.00000 & 2.93845 \\
27 & N4 & 6.92082 & 0.00000 & 3.95520 \\
28 & H7 & 6.69758 & -1.52032 & -8.75493 \\
29 & H9 & 13.20286 & -1.51919 & -5.19533 \\
30 & H11 & 13.01398 & -1.52035 & 1.99828
\end{tabular}

Nuclear repulsion energy $=848.549572$

SA-5-CASSCF $(12,11) / 6-31(+) G(d, p)$ energies for symmetry species 1:

root number $1 \mathrm{E}=-642.92121415$ a.u.

root number $2 \mathrm{E}=-642.85334432$ a.u.

root number $3 \mathrm{E}=-642.78157462$ a.u.

root number $4 \mathrm{E}=-642.77638550$ a.u.

root number $5 \mathrm{E}=\quad-642.74200577$ a.u.

MS-5-CASPT2/6-31(+)G(d,p) energies for symmetry species 1:

$\begin{array}{llllll}-644.90000415 & -644.82669014 & -644.76160557 & -644.74023737 & -644.72536889\end{array}$

SA-5-CASSCF $(12,11) / 6-31(+) G(d, p)$ energies for symmetry species 2: 
root number $1 \mathrm{E}=\quad-642.81668607$ a.u.

root number $2 \mathrm{E}=\quad-642.72887199$ a.u.

root number $3 \mathrm{E}=-642.69376654$ a.u.

root number $4 \mathrm{E}=-642.67304119$ a.u.

root number $5 \mathrm{E}=-642.66744092$ a.u.

MS-5-CASPT2/6-31(+)G(d,p) energies for symmetry species 2:

$\begin{array}{llllll}-644.75458113 & -644.68676649 & -644.64678503 & -644.62697839 & -644.61733244\end{array}$ 